\title{
Lineamientos para la construcción de un currículo crítico como base para la transformación desde la escuela
}

\author{
Guidelines for the construction of a critical curriculum as a basis for transformation from school
}

DOI: https://doi.org/10.21803/penamer.14.27.458

Rafael Sánchez Anillo https://orcid.org/0000-0002-8924-0558

¿Cómo citar este artículo?

Sánchez, R. (2021). Lineamientos para la construcción de un currículo crítico como base para la transformación desde la escuela. Pensamiento Americano, 14(27), 115-123

DOI: https://doi.org/10.21803/penamer.14.27.458

\section{Resumen}

Objetivo: El presente artículo de investigación se desarrolló con el objetivo de proponer unos lineamientos para la formación en autonomía desde un currículo crítico en estudiantes desde la escuela. Introducción: Los nuevos retos de la humanidad merece aportes desde la escuela y es precisamente desde estos lineamientos curriculares, una propuesta que desde la criticidad en las aulas propendan por una ciudadanía consciente, capaz de tener el conocimiento y preparación ciudadana para enfrentar las políticas de los currículos estandarizados. Resultados y conclusiones: que han alejado al ser humano de los valores éticos, morales, fraternales y solidarios ante los modelos educativos que alejan a los ciudadanos de los efectos de los procesos económicos y medioambientales. Método o metodología: basada en la investigación cualitativa desde un enfoque de investigación acción, fundamentado en la acción comunicativa permanente entre los diferentes actores del proceso educativo, debido a la crisis de la escuela ante las políticas impuestas desde los currículos ocultos que han permitido categorizar la calidad educativa con una mirada desde las competencias y logros. La información se obtuvo desde grupos focales de los diferentes miembros de los gobiernos escolares; de igual manera se realizó observaciones dentro y fuera del aula en 3 instituciones educativas.

Palabras clave: Currículo; Autonomía; Formación.

\begin{abstract}
Objective: This research article was developed with the objective of proposing guidelines for autonomy training from a critical curriculum in students from school. Introduction: The new challenges of humanity deserve contributions from the school and it is precisely from these curricular guidelines, a proposal from the criticality in the classrooms promotes a conscious citizenship, capable of having the knowledge and citizen preparation to face the policies of the standardized curricula results and conclusions they have alienated the human being from ethical, moral, fraternal and solidary values in the face of educational models that distance citizens from the effects of economic and environmental processes. Method or methodology: based on qualitative research from an action research approach, based on permanent communicative action between the different actors of the educational process, due to the crisis of the school before the policies imposed from the hidden curricula that have allowed to categorize the educational quality with a view from the competences and achievements. The information was obtained from focus groups of the different members of the school governments;
\end{abstract}


Similarly, observations were made inside and outside the classroom in 3 educational institutions.

Keywords: Curriculum; Autonomy; Training.

\section{Resumo}

a.

Palavras chave: a

\section{Perfiles}

Rafael Sánchez Anillo

Doctor en Educación. Magister en ciencias de la educación. Licenciado en matemáticas y física. Docente tiempo completo, Universidad del Atlántico. rafaelsanchez@ mail.uniatlantico.edu.co 


\section{Introducción}

La sociedad postmoderna que se vive en todas las latitudes del planeta tierra ha permitido reflexionar acerca de la educación que se imparte en la escuela desde la perspectiva de las políticas públicas de gobierno, la cual ha permitido hacer comparaciones epistemológicas entre la concepción de la educación para el hombre y hasta donde esta formación recibida permite desarrollar una verdadera autonomía en el estudiante desde contexto donde vive y se moviliza, partiendo desde la realidad histórica desde los griegos, fenicios y egipcios hasta transitar por las escuelas filosóficas epistemológicas de Frankfort, Kant, Russell, Rorty, Habermas y Freire entre otros.

Y desde una reflexión holística hacer una epistemología de la educación teniendo como referentes los fundamentos básicos de la ciencia, la tecnología y todo el desarrollo e innovación en pedagogía, metodología, didácticas, modelos educativos y curricular a través de los enfoques, tendencias, corrientes y modelos epistémicos, antropológicos, filosóficos, sociológicos, psicológicos y paradigmáticos, referente al aprendizaje significativo del educando desde el punto de vista de su capacidad de intervenir con autonomía en su entorno social de una manera crítica para solucionar los problemas que enfrenta cada día y transformar la realidad en beneficio de la humanidad.

En efecto, se acude a una sociedad con modelo económico capitalista donde la influencia de la ciencia, globalización y la tecnología en la sociedad del conocimiento ha conquistado diversos espacios de la vida: ha transformado nuestro modo de pensar, actuar y sentir; y por ende ha alterado aspectos fundamentales de lo cognitivo, lo axiológico y lo motor; por tanto, se asiste como espectadores a la destrucción de la ecología mundial para darle cumplimiento al gran poder industrial, comercial y tecnológico, en consecuencia todo se ve reflejado en el truncamiento de la evolución de algunas estructuras del cerebro relacionadas con el neocortex que a su vez se encuentra asociadas al interés emancipatorio. Así, se plantea en el currículo Integrado de Jurgo
Torres (2006). ¿Será que este modelo de sociedad es producto del modelo educativo impuesto por los gobernantes, o se encuentra inserto en el currículo oculto?

Las necesidades sociales, económicas e históricas del hombre han trascendido la cultura, desde la transculturación regional acontecida a través del tiempo o la aculturación producida a veces de manera repentina y apoyada, por ejemplo, por diversos elementos políticos y tecnológicos actuales que son inevitables y conducen a una contrariedad, primero al perder la identidad o desconocerla, y segundo al discriminar mediante el racismo, sexo, cultura hasta rechazar la pluralidad y diversidad cultural que impera en muchos países del mundo. A ello se le suma la profundización de las contradicciones y desigualdades sociales generadoras de conflicto y violencia.

Dentro del análisis de construcción del Plan Decenal de Educación de Barranquilla (2016-2026), se identificó las dificultades en la formación del estudiante en la utilización de las prácticas curriculares de docentes; de la misma forma se destacó la falta de comunicación entre los diferentes actores que componen el gobierno escolar y la no participación de la comunidad educativa en los manuales de convivencias en las instituciones educativas oficiales. En consecuencia, si no hay comunicación en la escuela entre los miembros de la comunidad es necesario abordar esta situación en esta investigación (Observatorio de Educación del Caribe Colombiano, 2016).

Ante este panorama se hace necesario proponer y discutir un currículo crítico que permita desde unos lineamientos factibles, formar estudiantes con autonomía que permita la toma de decisiones y en consecuencia tener la capacidad de discernir la problemática social, económica y política de su comunidad; es decir, prepararse para formar desde la escuela las próximas generaciones de ciudadanos y por ende las nuevas sociedades retadoras de la formación integral, con identidad y poder de decisión para enfrentar las situaciones y dificultades en la sociedad. Así se encuentra contemplado en el Plan Decenal 
del Distrito Barranquilla (2016-2026), el cual tiene grandes expectativas en la construcción de políticas públicas educativas que permitan la educación con calidad, integral y con autonomía.

Es pertinente considerar las premisas de Vigotsky y la construcción del aprendizaje desde la reflexión social que pueda favorecer la innovación curricular con el valor agregado, desde un enfoque curricular crítico, al ofrecer una estructura curricular que favorezca la transformación de las realidades (Torruño, 2020).

Esta investigación pretende desde los lineamientos curriculares construir autonomía en cada estudiante desde bases de un currículo crítico, visionadas desde la acción - participación de los actores escolares (alumnos, docentes, directivos, ex alumnos, padres de familia, instituciones religiosas y empresariales), desde donde emergen saberes producto de la aplicación de las técnicas e instrumentos de la investigación. El currículo es la oferta o propuesta educativa que implica un conjunto de acciones que la institución pone a consideración de los diversos públicos (padres de familia, estudiantes, docentes, directivos, administrativos, autoridades educativas y población en general) (Barraza, 2018).

Desde estos antecedentes, se plantea el siguiente interrogante ¿Cuáles serían las condiciones del corpus teórico, epistemológico y metodológico, que definen los lineamientos curriculares conducentes a la formación para la autonomía de ciudadanos críticos?

Por antecedentes enunciados, se formula el objetivo general: Proponer unos lineamientos curriculares coherentes con las condiciones de Corpus teórico, epistemológico y metodológico, conducentes a la formación en autonomía de ciudadanos críticos.

Es precisamente la formación en autonomía una propuesta que permitiría preparar a las nuevas generaciones para que de manera consciente y participativa intervengan en los procesos de transformación social desde una postura epistémica y dialógica en nuestra sociedad a través de posiciones críticas que permitan rechazar los modelos que elaboran los esquemas so- ciales que han permitido una debacle social y económica en América latina; no obstante, parafraseando a Correa (2004), desde la pedagogía poder evaluarse sin contaminación prejuiciosa emanada del mismo y de los demás en un acto consciente puede llegarse a un estado complejo de la conciencia para validar la acción más exitosa.

Al mismo tiempo, la tesis denominada "Concepto y Práctica del currículo de John Dewey”, de María del Coro Molinos Tejada (2002), quien hace una radiografía exhaustiva de la vida académica y los aportes de Dewey, que en la oscuridad aportó un gran baluarte a la educación desde sus posturas críticas y académicas a la pedagogía y al currículo.

Por lo anterior expresado, es obligación del educador comprometido con la autonomía proponer a sus estudiantes una pedagogía que construya la libertad y la crítica en los estudiantes, que permita enfrentar los modelos bancarios (Freire, 2012b). Coincidiendo con la construcción de un sujeto transformador el cual debe tener dimensiones de la perspectiva socio histórico cultural en vía de poner en práctica un desafío ético emancipatorio en la sociedad y desde esa formación que el sujeto se libera para ser autónomo (D’ Ángelo, 2004).

\section{METODOLOGÍA}

Desde la metodología planteada de un enfoque cualitativo, se escogió el diseño metodológico, más indicado: investigación - acción, Elliot (1990), Yuni y Urbano (2005), de tipo propositivo con un paradigma socio crítico; y sus técnicas: observación participativa, entrevista semi-estructurada, análisis documental y teórico, cursando todo el recorrido del espiral investigativo: rectificando, evaluando y validando desde grupos focales de padres de familia, ex-alumnos, docentes y directivos, para construir un aporte a la solución de crisis de autonomía en jóvenes, apoyados en la identificación de la debilidad de las prácticas curriculares en el acto educativo.

Iniciando desde el paradigma socio-crítico se permitirá priorizar las categorías originadas de la realidad que

Pensamiento Americano Vol. 14 - No. 27 -p.p. 115-123- · 2021 • Enero-Junio • Corporación Universitaria Americana • Barranquilla, Colombia • ISSN-e: 2745-1402 $\mathrm{http}$ //publicaciones.americana.edu.co/index.php/pensamientoamericano/index 
viven los miembros de la comunidad educativa, teniendo en cuenta que desde los actores en su dialéctica e interés, se identificarán los lineamientos curriculares que emergen para la solución de la carencia de autonomía en los estudiantes.

Deesta forma,lainvestigación-acción permitirárealizar una indagación introspectiva colectiva, emprendida por los sujetos pertinentes con el objeto de mejorar la racionalidad y justicia de las prácticas curriculares que inciden en la participación social, política y económica. Por consiguiente, reconociéndose los momentos de la investigación acción: diagnóstico, ejecución y evaluación. Se focalizaron 3 instituciones educativas (dos públicas y una privada), como sujetos de investigación con la particularidad de estudiantes en grado $8^{\circ}, 9^{\circ}, 10^{\circ}$ y $11^{\circ}$, los cuales pertenecen a familias con ingresos económicos diferentes en el Distrito Industrial y Portuario de Barranquilla (Colombia).

Partiendo de las categorías: autonomía, formación y currículo, descritas en los fundamentos epistemológicos y teóricos desde la estrategia metodológica a desarrollar que representa la apertura de un camino a la construcción de un saber epistémico y sistémico, se pretende aportar un nuevo conocimiento a las Ciencias de la Educación desde la emergencia que permite el constructo del saber educativo, desde lineamientos curriculares críticos que formen alumnos autónomos e integrales para enfrentar los cambios sociales y ambientales que ponen en crisis la sociedad.

Una vez finalizado la validación de instrumentos y sistematización con los miembros de la comunidad educativa, se procederá a elaborar los instrumentos y técnicas que se utilizarían en la Investigación-acción (entrevistas semiestructurada, observación participativay revisión documental) con sus respectivas categorías y subcategorías. Finalmente, su validación y sistematización de instrumentos y datos dentro del rigor metodológico para ser sometidos a las triangulaciones y análisis pertinentes.

\section{ANÁLISIS Y DISCUSIÓN DE LOS RESULTA- DOS}

En el objetivo específico 1. Se trianguló: revisión documental, entrevista semiestructurada y observación participante. Los análisis de resultados muestran la caracterización de currículos descontextualizados, sin evaluar por más de 3 años, poco socializado, y de la misma forma desconocido por padres y estudiantes.

De igual manera, se identificó un manual de convivencia desactualizado en 3 años, sin evaluación y con normas muy severas, desde sanciones y procedimientos como si fueran mayores de edad con aplicación de normas inconstitucionales, ocasionando violación de los derechos y sembrando semillas de injusticia en la escuela desde temprana edad en donde muchas veces finaliza con expulsión del estudiante, sin oportunidad del debido proceso. De la misma forma se evidenció el trabajo por asignaturas en el sector oficial; en contraste con el sector privado se identificaron contextualizados, interdisciplinarios, evaluados periódicamente con los actores educativos de la institución y con su entorno social.

En consecuencia, no hay organización institucional en la escuela del sector oficial y sus causas obedecen a las decisiones que se toman desde los directivos, sin socializar actuaciones administrativas y académicas que afectan la dinámica de un currículo que pueda generar a un estudiante con principios, valores y autonomía, daños emocionales e injustos, a diferencia del sector privado que se rigen por la planificación y administración del servicio educativo.

En el objetivo 2. Se trianguló observación participante en las categorías: formación, autonomía y currículo. La información mostró situaciones de las prácticas curriculares muy preocupantes, donde por voz de un estudiante, describe "Una clase de docentes con la pedagogía tradicional y sin ninguna didáctica”. De esta manera, se reclama una pedagogía y didáctica que facilite ser llamativo el acto pedagógico. No obstante, en la educación media solo se forma a los estudiantes a la mecanización de las pruebas saber para la consecución de obtener bachilleres pilos dejando a un lado la formación integral y la autonomía del estudiante. En secuencia, se develaron 2 categorías emergentes como es: la resiliencia y la identidad cultural desde la escuela como medio 
para transformar y humanizar la sociedad.

Estos antecedentes coinciden en que es necesario utilizar diversidad de instrumentos y procedimientos en la práctica docente y en los procesos evaluativos que permitan a los docentes hacer un seguimiento de su propia práctica, así como revisarse para reducir la distancia entre la teoría y la práctica docente (Verdeja, 2019).

Y para finalizar en el objetivo 3, se trianguló los saberes de teóricos científicos sociales reconocidos por sus aportes a las ciencias de la educación, Freire (Pedagogía del oprimido), Giroux (teoría de la resistencia social) y McLean (cerebro triuno), a través de un diálogo de saberes (Ver Tabla 1).
De manera significativa se destaca los conocimientos y saberes que emergen de esta triangulación y que le aportan a la pedagogía crítica importantes lineamientos para "la construcción de un currículo crítico como base para la transformación desde la escuela”.

Secuencialmente, en el análisis de resultado se puede observar una relación entre los diferentes teóricos que proponen la problematización, la reflexión, la acción, la práctica docente, el acto educativo desde la escuela y otros factores que inciden en ellas como: las capacidades emocionales, sensoriales y cognitivas, atribuidas a las funciones del cerebro reptiliano, límbico y neocórtex, que desde procesos y prácticas curriculares, se pueda estimular y lograr la potenciación máxima del cerebro para desarrollar una criticidad, transformación de la sociedad,

Tabla 1.

Triangulación de hallazgos teóricos

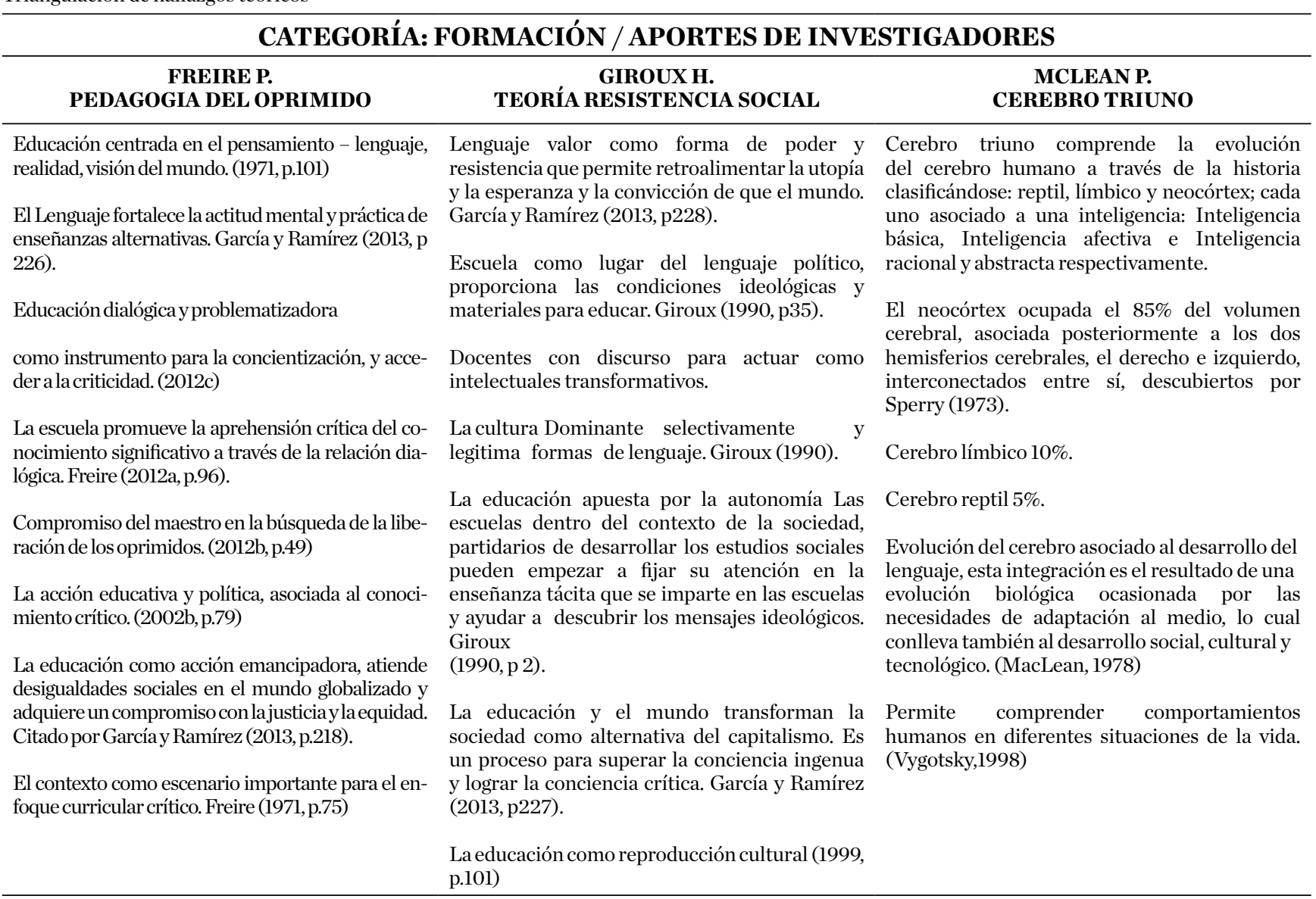

Fuente: Elaboración propia 
desarrollo cultural y tecnológico, siendo la escuela "un lugar del lenguaje político, que proporciona las condiciones ideológicas y materiales para educar" Giroux (1990, p. 35).

Finalmente, otro elemento relevante que surge se refiere a la identidad cultural, dándole una explicación genética desde las funciones del cerebro reptil encargado de la transmisión de conocimientos de generación a generación. En este sentido se constituye la identidad cultural como un proceso educativo que debe valorar la diversidad de las memorias históricas del continente, según Freire (2012b).

En consecuencia, las prácticas curriculares deben responder a las condiciones de un sujeto neurobiológico, emocional, histórico, social, cognitivo, cultural y político para que incida en la transformación de las sociedades alcanzando su autonomía.

\section{CONCLUSIONES}

Los objetivos específicos correlacionados con fundamento en la pregunta problema de esta investigación permitió cumplir con los fines establecidos para aportar solución a las dificultades de la formación en autonomía visionada desde las prácticas curriculares, las cuales nos arrojaron las siguientes conclusiones:

1. Las prácticas curriculares se encuentran centradas en la formación del estudiante en el rendimiento del índice sintético de calidad, distanciándolo de la formación integral e inmersa en la educación bancaria, anunciada por Freire (2012b): "Las prácticas curriculares están descontextualizadas y se necesita implementar acciones y políticas institucionales para contextualizar el sistema educativo".

2. No existe hilo de comunicación a través del lenguaje que permita la construcción de un currículo comunicativo, ya que los distintos actores del sistema educativo oficial no consensúan sus decisiones y acciones, contribuyendo a un caos administrativo y académico, y por ende no se visiona en la práctica curricular la interdisciplinariedad, sin embargo, en el sector educativo privado se construyen los proceso por decisiones administrativas y de planificación, con los diferentes componentes del gobierno escolar.

3. El sistema educativo oficial tiene niveles bajos de dialogicidad, concienciación, problematización, reflexión y acción en el acto educativo que provoque la praxis social, ya que en estos procesos inciden en factores como: las emociones, capacidades cognitivas y sensoriales, atribuidas a la funcionalidad del cerebro. Estos lineamientos a fines a la pedagogía crítica está fundamentalmente interesada en la comprensión de la relación entre el poder y el conocimiento, que facilite la preparación de los estudiantes para que se desempeñen en la sociedad, ya sea de dominación o subordinación, es decir, poner en práctica la funcionalidad de un currículo crítico (Sánchez et al, 2018).

4. La escuela oficial no construye un manual de convivencia con los actores del gobierno escolar, situación que permite la aplicación de normas inconstitucionales y violatorias a los menores y adolescentes de manera injusta. Mientras que las instituciones privadas desarrollan la participación, socialización y construcción del reglamento de estudiantes, docentes y padres de familia.

5. Finalmente, se aborda la crisis de la escuela desde los lineamientos curriculares en la inclusión de la resiliencia e identidad cultural, solicitados por los estudiantes y padres de familia para transformar y humanizar la sociedad.

\section{RECOMENDACIONES}

Construir participativamente lineamientos curriculares con condiciones del corpus teórico, epistemológico y metodológico, que defina los fun- 
damentos curriculares conducentes a la formación para la autonomía a ciudadanos transformadores de la cultura política y social, en el proceso enseñanza aprendizaje y desde las prácticas curriculares estimulan el cerebro y sus predominios para alcanzar una autonomía crítica, dialógica y transformadora, donde la estructura cerebral y su conectividad pueda cambiar con la experiencia diaria su autonomía, lo cual se refleja en su relación consigo mismo, su entorno y la sociedad, de esta forma se recomienda:

- Que las prácticas curriculares esté en constante acción comunicativa a través del diálogo, para sustentar los lineamientos curriculares como construcción social donde el lenguaje sea un elemento fundamental que permita encontrar nuevos significados e interpretaciones, el cual estructure una subjetividad socio -histórico - cultural como práctica comunitaria liberadora y transformadora de la realidad social.

- La escuela debe estar centrada en la participación y construcción colectiva del conocimiento como centro de formación de conciencia política. Es primordial un currículo y manual de convivencia que sea negociado con la comunidad escolar en constante interacción dinámica en la acción y la reflexión con el compromiso profesional de los educadores.

- Inclusión de lineamientos curriculares desde actividades y asesorías pedagógicas en la resiliencia e identidad cultural que permita transformar y humanizar la sociedad.

- La escuela, como escenario para poner en práctica las tres funciones esenciales encomendadas por la sociedad: transmisión cultural, integración social y socialización. Desde la interdisciplinariedad que integre campos de conocimientos y experiencias que faciliten una comprensión más reflexiva y crítica de la realidad social.

\section{Referencias}

Barraza, N. (2018). El Currículum, Análisis y Reformulación del Concepto. Dictamen Libre, 1(22), 113-118. doi: https://doi org/10.18041/2619-4244/dl.22.5032

Correa, C. (2004). Currículo Dialógico, sistémico e interdisciplinar. Editorial Magisterio.

D’ Ángelo, O. (2004). Autonomía integradora y transformación social: El desafío ético emancipatorio de la complejidad. CIPS, Centro de Investigaciones Psicológicas y Sociológicas

Elliot, J. (1990). La investigación - acción en Educación. Edición Morata.

Freire, P. (1971). Conciencia crítica y liberación. Editorial Camilo

Freire, P. (2012a). La educación en la ciudad. Editorial Siglo XXI.

Freire, P. (2012b). Pedagogía del oprimido. Editorial Siglo XXI.

García, L. \& Ramírez, M. (2013). Pedagogía crítica y comunicaciones: Una mirada sobre los jóvenes escolares. Comunic@red1(1).217-244.

Giroux, H. (1990). Los profesores como intelectuales. Paidós.

MacLean, P. (1978). The Triune Brain Evolution. Plenun Press. México: Grijaldo.

Molinos M. (2002). Concepto y Práctica del currículo en John Dewey. Ediciones Universidad de Navarra, Pamplona (4). 416. https://doi.org/10.15581/004.4.206

Observatorio De Educación del Caribe Colombiano. (2016, 2 de agosto). En Barranquilla la Educación está de Primera. https://www.uninorte.edu.co/web/blogobservaeduca/ blogs/-/blogs/en-barranquilla-la-educacion-esta-deprimera

Sánchez, N.,Sandoval,E., Goyeneche, R., Gallego, D \& Aristizábal, L. (2018). La pedagogía crítica desde la perspectiva de Freire, Giroux, y McLaren: su pertinencia en el contexto de Colombia y América Latina. Revista Espacio 39(10). 41.

Pensamiento Americano Vol. 14 - No. 27 -p.p. 115-123- · 2021 • Enero-Junio • Corporación Universitaria Americana • Barranquilla, Colombia • ISSN-e: 2745-1402 http://publicaciones.americana.edu.co/index.php/pensamientoamericano/index 
Torres, J. (2006). Globalización e interdisciplinariedad: El currículo integrado. Ediciones Morata.

Toruño, C. (2020). Aportes de Vigotsky y la pedagogía crítica para la transformación del diseño curricular en el siglo XXI. Innovaciones Educativas, 22(33), 186-195. https://doi. org/10.22458/ie.v22i33.3043

Verdeja, M. (2019). Concepto de educación en Paulo Freire y virtudes inherentes a las orientaciones para una escuela intercultural. Contextos: Estudios de humanidades y ciencias sociales 42. 7-7.

Vygotsky, L. (1998). Teorías Vygotsky y Piaget: Desarrollo del niño y del Adolescente

Yuni, Y \& Urbano, C. (2005). Mapas y herramientas para conocer la escuela: investigación. Etnográfica e investigación-acción. 
